Planetary Systems in the Universe - Observation, Formation and Evolution

Proceedings IAU Symposium No. 202, (c)2004 IAU

Alan Penny, Pawel Artymowicz, Anne-Marie Lagrange, \& Sara Russell, eds.

\title{
A Deep Optical Search for Substellar Candidates in the Nearby $\rho$ Ophiuchi Star-Forming Region
}

\author{
S. Terebey, and D. Van Buren
}

Extrasolar Research Corp, 2569 Balltown Rd, Niskayuna NY 12309 USA

T. H. Jarrett

Caltech, IPAC 100-22, Caltech, Pasadena CA 91125 USA

\section{T. N. Gautier}

Jet Propulsion Lab, MS 169-327, 4800 Oak Grove Dr, Pasadena CA 91109 USA

\begin{abstract}
We report preliminary results from a deep I-band optical search for substellar candidates in the $\rho$ Oph star-forming region. The dusty molecular cloud provides an opaque screen at optical wavelengths, making the survey sensitive to faint substellar candidates near the cloud surface. Of the $89 \mathrm{ob}-$ jects detected in the oph $\mathrm{A}$ and oph $\mathrm{C}$ molecular cores, one third are substellar candidates.
\end{abstract}

\section{Introduction}

Theoretical models predict giant planets and brown dwarfs are hottest and brightest when young (Burrows et al. 1997), thus motivating searches for substellar objects in star-forming regions and star clusters. Deep optical and NIR searches have been effective in finding substellar candidates, with recent surveys detecting objects down to approximately $10 \mathrm{M}_{J u p}$ or $0.01 \mathrm{M} \odot$ (Zapatero Osorio et al. 2000). This mass regime begins to probe the transition between giant planets and brown dwarfs, and will provide important constraints on how such objects form.

The $\rho$ Oph molecular cloud is nearby $(160 \mathrm{pc})$, young ( $\sim 1 \mathrm{Myr})$, has high extinction, and has a high level of star-formation activity. Previous nearinfrared surveys of $\rho$ Oph (Comeron et al. 1993, 1998; Luhman \& Rieke 1999) have identified a number of substellar candidates which have been confirmed by follow-up up spectroscopy (Luhman, Liebert, \& Rieke 1997; Wilking, Greene, \& Meyer 1998; Cushing, Tokunaga, \& Kobayashi 2000).

We report preliminary results from a deep survey to detect substellar objects in the $\rho$ Oph star-forming region. In order to detect substellar candidates with high efficiency our approach was to take sensitive large-format images of the $\rho$ Oph cloud, using the cloud itself as an opaque source which effectively screens out background objects. The resulting survey is sensitive to masses as low as 5 $\mathrm{M}_{J u p}$ near the surface of the $\rho$ Oph cloud. 


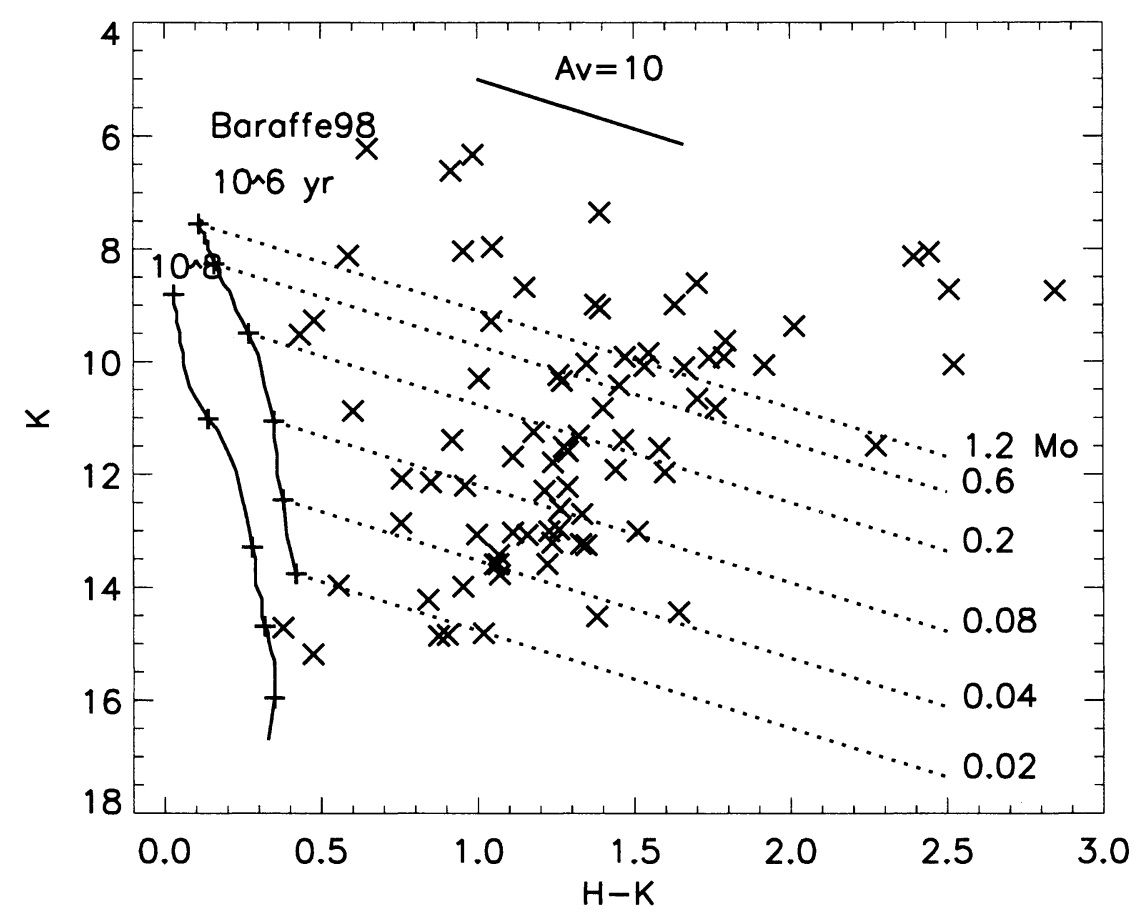

Figure 1. A NIR color-magnitude diagram. The deep I band images are remarkably efficient at detecting substellar candidates, with $34 \%$ of the sample lying below the $0.08 \mathrm{M} \odot$ substellar boundary. The substellar candidates include GY141, a young brown dwarf based on its M8.5 spectral type and low-surface gravity features (Luhman et al. 1997).

\section{Observations}

We obtained wide-format (13.6 ${ }^{\prime}$ FOV) I-band images of three fields in the $\rho$ Oph core in June 2000 using the COSMIC array camera on the $5 \mathrm{~m}$ Hale Telescope at Palomar Observatory. Results are presented for two fields, the Oph A and Oph $\mathrm{C}$ cores. The extinction is high, about $\mathrm{A}_{V}=20\left(\mathrm{~A}_{I}=10, \mathrm{~A}_{K}=2\right)$ over most of each field so that possible background sources appear highly reddened. The estimated number of foreground sources may approach four, using a $0.1 \mathrm{pc}^{-3}$ space density of field objects and $20 \mathrm{pc}^{3}$ foreground volume per COSMIC field.

\section{Discussion}

In the two fields we extracted a total of 89 sources down to the approximate magnitude $\mathrm{I}=24.5$ detection limit. Sources are brighter in the near-infrared due to the lower extinction at those wavelengths, so we cross-compared our data with the Two Micron All Sky Survey (2MASS) as a means to quickly identify 
interesting targets. Most of the I band sources had 2MASS counterparts. In fact, $38 \%$ of the 2 MASS sources within the two fields are detected at I band, thus showing the I band survey samples the cloud to substantial depth. In addition, there are six I band sources which do not have 2MASS counterparts. We do not discuss these sources further except to note they represent either $M<15 M_{J u p}$ substellar objects or background sources near the cloud edge.

Figure 1 shows a NIR color magnitude diagram to identify substellar candidates, in which we plot the I band sources which have 2MASS counterparts. Theoretical evolutionary tracks are shown as solid lines for 1 and $100 \mathrm{Myr}$ isochrones (Baraffe et al. 1998). Dashed lines show how extinction due to standard ISM dust affects objects of different mass. The lack of sources in the lower right is not significant but shows that, as expected, faint highly obscured objects are not detected at I band. The NIR error bars are small (0.02 mag) but grow to about 0.20 in $\mathrm{H}-\mathrm{K}$ near the $\mathrm{K}=15$ limiting magnitude.

Spectra exist for most of the brighter objects having $\mathrm{K}<12$ and show these objects are primarily young stellar objects within the cloud (Luhman et al. 1999). A high fraction of about $34 \%$ of our I band sample are substellar candidates, falling below the $0.08 \mathrm{M} \odot$ substellar boundary. Of the four candidates in common with other surveys, follow-up spectroscopy confirm three as substellar with estimated masses below $40 \mathrm{M}_{J u p}=0.04 \mathrm{M} \odot$ (Luhman et al. 1997; Cushing et al. 2000).

Future work will include spectroscopy to determine the nature of the substellar candidates. Coordinated millimeter observations are underway to search for the presence of associated gas and dust. The presence or absence of circumstellar gas and dust in this young $(\sim 1 \mathrm{Myr})$ star-forming region will provide strong clues to the age and origin of the substellar candidates.

Acknowledgments. This publication makes use of data products from the 2MASS Survey, a joint project of the U. of Massachusetts and IPAC/Caltech, funded by NASA and the NSF.

\section{References}

Baraffe, I., Chabrier, G., Allard, F., \& Hauschildt, P. H. 1998, A\&A, 337, 403

Burrows, A., Marley, M., Hubbard, W. B., Lunine, J. I., Guillot, T., Saumon, D., Freedman, R., Sudarsky, D., \& Sharp, C. 1997, ApJ, 491, 856

Comeron, F., Rieke, G. H., Burrows, A., \& Rieke, M. J. 1993, ApJ, 416, 185

Comeron, F., Rieke, G. H., Claes, P., Torra, J., \& Laureijs, R. H. 1998, A\&A, 335, 522

Cushing, M. C., Tokunaga, A. T., \& Kobayashi, N. 2000, AJ, 119, 3019

Luhman, K. L., Liebert, J., \& Rieke, G. H. 1997, ApJ, L165

Luhman, K. L., \& Rieke, G. H. 1999, ApJ, 525, 440

Wilking, B. A., Greene, T. P., \& Meyer, M. R. 1998, AJ, 117, 469

Zapatero Osorio, M. R., Béjar, V. J. S., Martín, E. L, Rebolo, R., Barrado y Navascués, D., Bailer-Jones, C. A. L., \& Mundt, R. 2000, Science, 290, 103 